\title{
Wireless Body Area Networks Node Sleep Strategy Based On Compressed Sensing and Sparse Representation \\ Jiashun Chen ${ }^{1,3, a}$, Yuebin Zhou ${ }^{2,3, b}$ and Tao Wang ${ }^{1, c}$
}
1. College of Mechanical Automation, Wuhan University of Science and Technology, Wuhan 430081, China;
2. School of Mechanical and Auto Engineering, Hubei University of Arts and Science, Xiangyang 441053, China;
3. Collaborative Innovation Center of Hubei Province for Auto Parts Manufacturing Equipment Digitization Xiangyang 441053, China

a741479160@qq.com, bybonly@163.com, wangtaw@163.com

\begin{abstract}
Keywords: WBAN; sleep strategy; compressed sensing; sparse representation
Abstract: Aiming to the problem that the battery energy of the node is limited and not easy to be replaced in WBAN, this paper proposes a new node sleep strategy based on compressed sensing and sparse representation theory (NSS-CS), which using the compressed sensing theory to compress the test samples, and adopting the sparse representation theory to recognize them. When the physiological signals collected by the nodes are in the normal range, NSS-CS can identify those normal signals, stop nodes to transmit normal signals, and make nodes convert into a sleep state to prolong the sleep time of nodes and reduce the amount of transmitting data, thus the node energy consumption can be reduced. Using NS2 software, the simulation of node delay and energy consumption have been implemented, the simulation results show that, WBAN physiological signals are usually in a stable range, compared with the traditional TDMA and BCMAC protocols, the NSS-CS can effectively reduce the energy consumption and delay.
\end{abstract}

\section{Introduction}

Wireless Body Area Network (WBAN) is a kind of network, using sensor nodes on or inside the human body to collect human physiological data, like body temperature, blood pressure, and other physiological signals. Generally, nodes are powered by battery, which is very limited, and is not easy for replacing in the human body. So researching on the method of nodes energy saving has important practical significance in WBAN [1].

Compressed Sensing (CS) theory often includes the compression sampling, observations, and reconstruction, and in recent years, it has been widely used in the WBAN [2,3]. In 2009, the sparse representation theory was first applied in the classification and the sparse representation based classifier (SRC) was proposed, which made the pattern recognition problem converted to the signal sparse representation problem and provided the theoretical basis for the signal recognition [4]. In medical tests, many physiological signal databases have been established, which provide reliable sample information for sparse representation. Such as MIT-BIH is provided by the MIT to study arrhythmia, and Wearable Action Recognition Database (WARD) is provided by the University of California Berkeley to study human daily action. Those databases make the SRC widely used in WBAN.

Most signals collected by nodes are normal signals in WBAN, and these signals are not necessary to be transmitted. Transmitting signals without distinction are undoubtedly a waste of energy. This paper proposes a new node sleep strategy based on CS theory and SRC (NSS-CS) to compress and classify signals, make nodes stop transmitting the normal signal and entering into sleep state, thus the sleep time of the node is increased and the energy consumption of the node is effectively reduced. 


\section{Node Sleep Strategy Analysis}

The node of wireless sensor network generally includes sensor, central processing unit (CPU) module, and radio module, and the energy consumption of each module is shown in Fig.1 [5]. The mainly energy consumption concentrates on data transmitting and receiving status of the radio module, so reducing the data transmitting and receiving time of the radio module can effectively reduce the energy consumption. The multiple access control (MAC) protocol directly controls the radio module and has a large effect on the energy consumption of the node. Generally the MAC protocol mainly adopts two ways to reduce the energy consumption of the node, one is to increase the sleep time of the radio module, and the other is to avoid the conflict of data transmission.

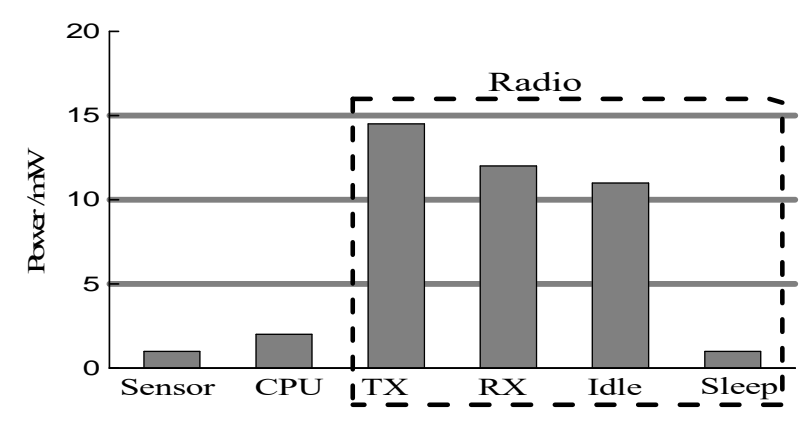

Fig. 1 Energy consumption of sensor nodes

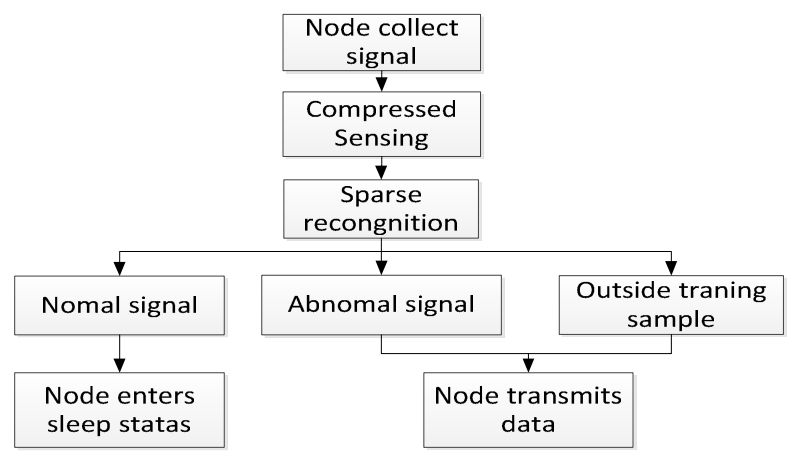

Fig.2 Signal compression and classification

The physiological signal databases provide reliable training samples for sparse representation of the test sample. The signals collected by nodes are usually in a normal range and have corresponding training samples in the database. The NSS-CS algorithm can control the nodes sleep status. The specific process of the signal compression and classification in the node is showed in Fig.2. When WBAN is started, the node begins to collect the signal as a test sample. Then the test sample is compressed by CS and recognized by SRC. If the recognition result shows that the test sample is a normal signal, the node enters sleep state, if the test sample is an abnormal signal or outside training samples, the node transmits data to the center node.

\section{Realization of NSS-CS Algorithm}

Random Projection. Random projection (RP) makes the data from higher dimensions into the lower dimensions. If $x \in R^{n \times 1}$ is a test sample, $x$ can be projected into a $m$-dimensional subspace via $\Phi \in R^{m \times n}(m<n)$.

$$
\grave{1}_{m \times 1}=\Phi_{m \times n} x_{n \times 1} .
$$

Selecting a suitable RP matrix is very essential for the compression and reconstruction of the test sample [6]. In order to make sure that $x_{n \times 1}$ is recovered from $x_{m \times 1}$, the $\Phi$ needs to satisfy the restricted isometry property (RIP). The $\Phi$ satisfies the RIP, and there exists a constant $\delta_{s} \in(0,1)$ :

$$
\left(1-\delta_{s}\right)\|x\|_{2}^{2} \leq\left\|\Phi_{\Lambda}\right\|_{2}^{2} \leq\left(1+\delta_{s}\right)\|x\|_{2}^{2}
$$

In Eq.2, $\Lambda \subset\{1, \mathrm{~L}, n\},|\Lambda| \leq s$, and $\Phi_{\Lambda}$ is the submatrix of $\Phi$ which is constituted by the related columns of the index $\Lambda$ in $\Phi$. $s$ is the number of signal sparsity.

The Sub-Gaussian random matrix has been proved to satisfy the RIP condition with high probability [7]. The structure of Sub-Gaussian random matrix is very sparse, which makes the storage space and calculation complexity of the projection data can be greatly reduced, 
Sub-Gaussian random matrix is very suited to be used in WBAN of limited resources.

Establish Over-Complete Dictionary. Each node is distributed in different locations of the body and collects the different types of physiological signals. First, each training sample in the physiological database is classified as a normal and abnormal signal, and each is numbered. Assuming that each physiological signal contains $w$ types, and then all types are selected from the database. Let $d_{i} \in R^{k_{i}}$, and $d_{i}$ represents the $i$-th type of physiological signal in the database. Each type contains $k$ training samples. The dimension of each training sample is $m$. Let $a_{i k i} \in R^{m}$, $a_{i k_{i}}$ represents the $k$-th training sample in the $i$-th type. Then, let $d_{i}=\left[a_{i 1}, a_{i 2}, \ldots, a_{i k_{i}}\right]$, and make the $w$ kinds of physiological signals constitute the over-complete dictionary $D$.

$$
D=\left[d_{1}, d_{2}, \mathrm{~L}, d_{w}\right]=\left\lfloor a_{1,1}, a_{1,2}, \mathrm{~L}, a_{w, n_{w}}\right\rfloor, D \in R^{m \times n} .
$$

$n=n_{1}+n_{2}+\mathrm{L}+n_{w}$, and $D$ is directly constructed from the training samples. In order to reduce the amount of dictionary data volume, the Sub-Gaussian random projection matrix is used to reduce the dimension of $D$ and improve the speed of compression and classification.

$$
\stackrel{1}{D}_{L \times n}=\Phi_{L \times m} D_{m \times n}(L<m) .
$$

$L$ is the dimension of $\vec{D}$, so the dimension of $D$ is reduced from $m$ to $L$.

Sparse Representation of Test Samples. If $y$ is the test sample collected by the node, it also needs to reduce the dimension via the Sub-Gaussian random projection matrix. So $y_{L \times l}=\Phi_{L \times m} y_{m \times l}$. The coefficient $x$ of the test sample $y_{i}$ is calculated by sparse representation theory in Eq.5.

$$
\hat{x}=\arg \min \|x\|_{0}, \text { subject to } \hat{y}=\grave{D} x \text {. }
$$

\|\|$_{0}$ is $l_{0}$-norm, and $\|x\|_{0}$ represents the number of non-zero elements in $x$. According to CS theory, if $x$ is sparse enough, minimize $l_{0}$-norm can be converted into the $l_{1}$-norm. The data collected by the node often contains noise, so $\varepsilon$ is added as an error term.

$$
\hat{x}=\arg \min \|x\|_{1}, \text { subject to }\|\hat{y}-\grave{D} x\|_{2} \leq \varepsilon .
$$

Identification of Valid Samples. Some abnormal test signals which are not in training samples may not be recognized, in order to prevent these signals to be omitted, the sparse concentration Index (SCI) is used to judge the test samples before the physiological signal classification.

$$
\operatorname{SCI}(x)=\frac{n \cdot \max _{i}\left\|\delta_{i}(x)\right\| /\|x\|_{1}-1}{n-1} \in[0,1] .
$$

$n$ is total the type number of training samples, $i=1,2, \ldots, n . \delta_{i}(x)$ is the coefficient of the $i$-th place in $\hat{x}$. If $\operatorname{SCI}(\hat{x})=1$, the test sample can be represented by only a training sample of one type. If $\operatorname{SCI}(\hat{x})=0$, the test sample is not in the training sample. So a threshold $\tau, \tau \in[0,1]$ needs to be set. If $S C I(\hat{x}) \geq \tau$, the test sample is the valid sample, and otherwise it is the invalid sample. The Invalid sample as an abnormal signal is transmitted to the center node. If the test sample is a valid sample, the test sample needs to be further determined whether it is normal.

Switching Nodes Sleep State. Due to the noise impact, the non-zero elements will be distributed between many types, the residual classification function is used to identify the test sample. 
$\min r_{i}(\hat{y})=\left\|\hat{y}-\grave{D} \delta_{i}(\hat{x})\right\|_{2}$.

$i=1,2, \ldots, n$., andidentity $(y)=\operatorname{argmin}_{i} r_{i}(y)$, identity $(y)$ represents the $i$-th tag numcber of the minimum residual, and that is the final classification results. The flow chart of NSS-CS algorithm is shown in Fig.3.

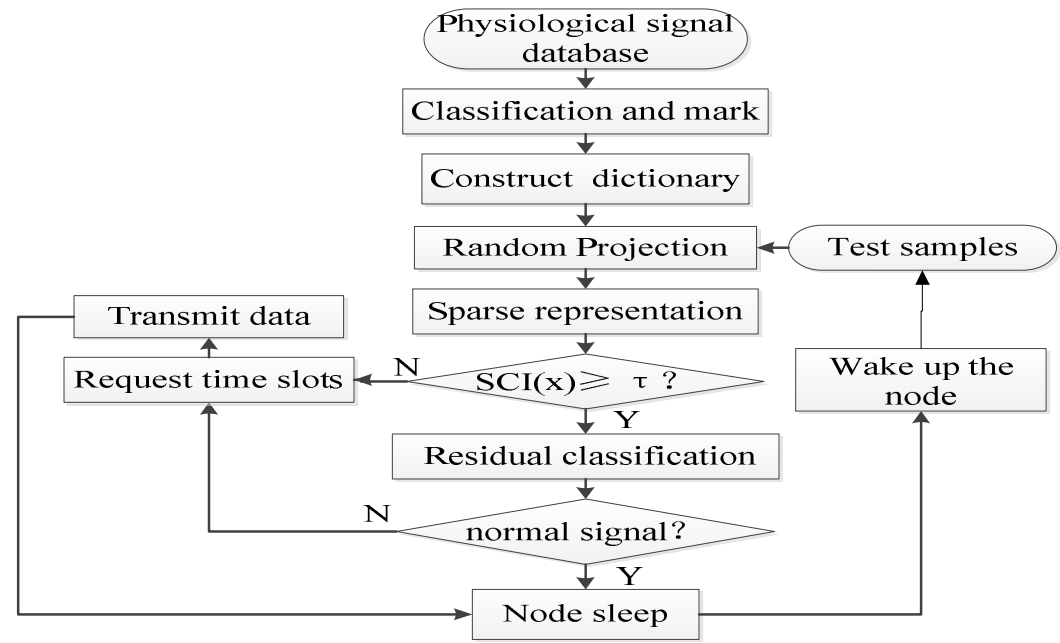

Fig.3 NSS-CS algorithm flow chart

First the $\tau$ and the $\varepsilon$ are set, and each training sample in the database is classified and numbered, then the over-complete dictionary $D$ and the test sample $y$ is constructed, then $D$ and $y$ are projected to a low-dimensional space via a random matrix $\Phi$, and CPU calculates the value of $\hat{x}$ via sparse representation theory and $\operatorname{SCI}(\hat{x}) . \operatorname{SCI}(\hat{x})$ is compared with the $\tau$, if $y$ is an invalid sample, the node requires time slots to the center node, otherwise, $y$ needs to be further identified by residual classification function. The number $i$ of the identification result is compared with tag number in training sample to judge whether $y$ is the normal signal. If $y$ is the normal signal, the node immediately entered the sleep state and waits to be woken up next time. However, if $y$ is not the normal signal, the node transmits data as well as the invalid sample to the center node.

Algorithm Validation. As shown in Eq.9, $C R$ is compression ratio, $N$ is the original signal dimension, and $M$ is the dimension of the compressed signal.

$$
C R=\frac{N-M}{N} \text {. }
$$

In order to verify the validity and scope of the NSS-CS, a simulation experiment is completed, adopting Sub-Gaussian random matrix to reduce the dimension of test sample and dictionary, taking $\tau=0.5$ and $\varepsilon=0.03$. Using ECG signals in MIT-BIT as training samples, the training sample dimension is $m=500$, and all training samples are constructed over-complete dictionary $D \in R^{500 \times 2000}$. ECG signals of 10 patients with different types of heart rate have been collected in a hospital, and each ECG signal is used as the test sample $y$. The average recognition rate and the classification time of ECG signals under different $C R$ via NSS-CS are shown in Table 1.

Table 1 Average recognition rate and the classification time of ECG signals under different $C R$

\begin{tabular}{ccc}
\hline $\mathrm{CR}$ & average recognition rate (\%) & classification time $(\mathrm{ms})$ \\
\hline $\mathrm{CR}=0$ & 100 & 276 \\
$\mathrm{CR}=0.3$ & 99.8 & 195 \\
$\mathrm{CR}=0.5$ & 99.2 & 142 \\
$\mathrm{CR}=0.7$ & 98.2 & 104 \\
$\mathrm{CR}=0.9$ & 97.1 & 72 \\
\hline
\end{tabular}


As shown in Table 1 , while $C R=0.1,0.3,0.5$, the average recognition rate is decreased slowly, but the classification time is significantly reduced, and increasing $C R$ can effectively reduce the classification time. When $C R=0.7$ and 0.9 , the average recognition rate decreases faster, the signal will be more mistakenly identified, so choosing the appropriate $C R$ can make signal recognition rate higher and delay time smaller.

\section{Energy Consumption Analysis and Simulation}

Analog to Information Conversion (AIC) is a new method to sample data based on CS theory, and the sensor can directly sample and compress the data by the AIC [8]. But at present, most sensors use Analog Digital Conversion (ADC) mechanism to sample data based on Nyquist-Shannon sampling theorem. Therefore, NSS-CS only compressed and classified data which is sampled by the $\mathrm{ADC}$ and the data which needed to be transmitted is still not compressed data.

As shown in Fig.1, the node energy consumption is mainly in sensors, CPU, transmitting data, receiving data, idle listening, and sleeping. The energy consumption can be expressed as Eq.10.

$$
E=E_{s}+E_{C P U}+n\left(E_{t}+E_{r}\right)+E_{i}+E_{\text {sleep }} .
$$

$n$ is the working times of node transceiver, $E_{s}$ is the sensor energy consumption, $E_{C P U}$ is the CPU energy consumption, $E_{t}$ is the transmitting data energy consumption, $E_{r}$ is the receiving data energy consumption, $E_{i}$ is the idle energy consumption, and $E_{\text {sleep }}$ is the sleeping energy consumption.

TDMA and CDMA are two basic MAC protocols that commonly are used in WBAN. The TDMA protocol avoids data collision and retransmission, distributes independent slots for each node, does not need too much control information for data transmission, and makes the node timely enter the sleep state. So the TDMA mechanism is more appropriate to implement energy efficiency analysis in WBAN. $E_{i}$ and $E_{\text {sleep }}$ can be ignored under the TDMA mechanism.

$$
E=E_{s}+E_{C P U}+n\left(E_{t}+E_{r}\right)
$$

$E_{s}$ and $E_{C P U}$ are small, so the main factor of the energy consumption in the node is $n$.

Network Simulator Version2 (NS2) is open source code simulation software; the source code can be modified to set some parameters to simulate the network layer and data link layer in WBAN. Because the number of nodes that collected the abnormal signals are less and the data transmission distance is shorter in WBAN, so the star topology is used in the simulation experiment. The simulation parameters are shown in Table 2.

Table 2. Simulation parameters in WBAN

\begin{tabular}{cc}
\hline simulation parameters & parameter values \\
\hline Simulation time $(s)$ & 1200 \\
Number of nodes & 21(Includes a center node) \\
Maximum transmission radius $(m)$ & 1.5 \\
Initial energy $(m J)$ & 100 \\
Transmission power $(u W)$ & 24 \\
Reception power $(u W)$ & 20 \\
Sleeppower $(n W)$ & 100 \\
\hline
\end{tabular}

While processing the same data packet by difference MAC protocols, the energy consumption of sensor and CPU is almost the same. The BCMAC is a MAC protocol based on TDMA mechanism [9]. NSS-CS can stop the node to transmit normal signals, so the transceiver times are less than the traditional TDMA protocol and BCMAC protocol.

When the signal is normal, the traditional TDMA protocol needs the node to maintain listening 
state if no data to be transmitted, but the BCMAC protocol doesn't. So the traditional TDMA protocol consumes more energy than BCMAC protocol. However, when the signal is normal, NSS-CS directly makes the node enter a sleep state, so the energy consumption is less than the traditional TDMA protocol and BCMAC protocol. The energy consumption simulation results of a WBAN node under the traditional TDMA, BCMAC and NSS-CS are shown in Fig.4.

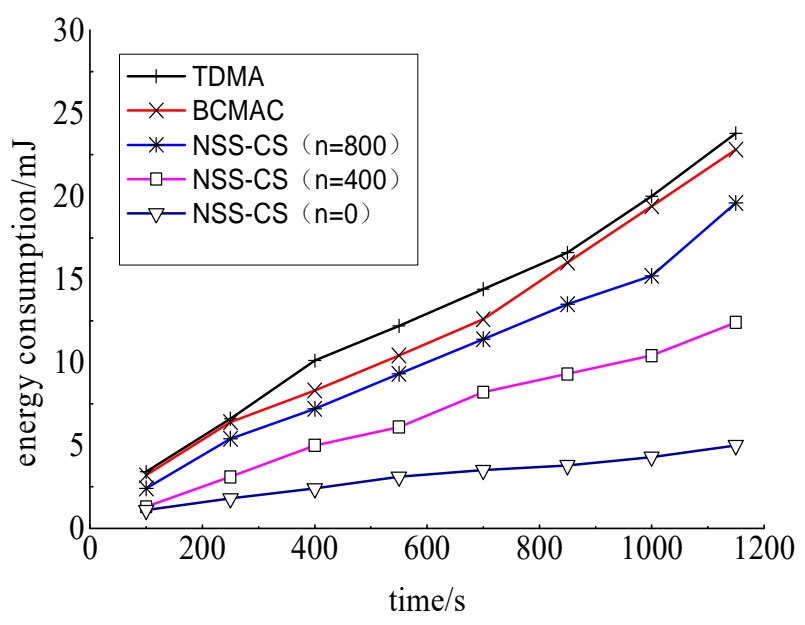

Fig.4 Node energy consumption simulation

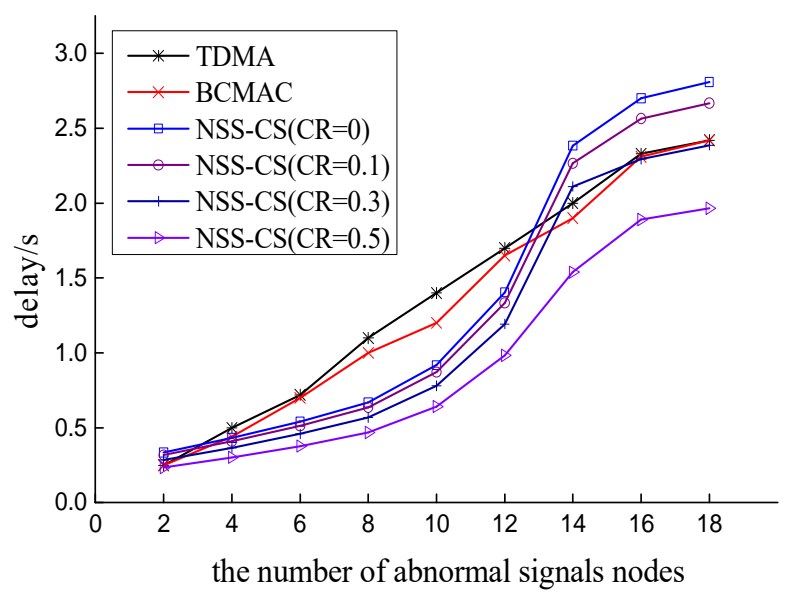

Fig.5 Delay comparison chart

When $n=0$, the signal collected by the node is the normal, NSS-CS stops the node transmitting the normal signal. The energy consumption using of NSS-CS is less than the traditional TDMA and BCMAC protocols.

Delay Analysis and Simulation. Collecting the same data, the sensor costs the same time and the time of processing data can be ignored in the traditional TDMA protocol and the BCMAC protocol. However, the data in the dictionary are very large, the time of compressing and classifying signals cannot be ignored in NSS-CS. Time slots of the traditional TDMA protocol and the BCMAC protocol are fixed in the two adjacent data transmission, So whether the signal of node collecting is normal or not, the delay of data transmission is:

$$
T_{1}=n \cdot S
$$

$S$ is a fixed time slot, and $n$ is the number of all nodes, which is known from Table. 2 and $n=20$. The NSS-CS can make nodes collecting normal signals into sleep status, so the delay of the NSS-CS is related to the number of abnormal nodes in WBAN.

$$
T_{2}=k \cdot S+k \cdot \widetilde{C}_{t}
$$

$k$ is the number of nodes collecting abnormal signals in WBAN, $0 \leq k \leq n$, and $\widetilde{C}_{t}$ is the average time that the node consumed in compressing and classifying the test sample. Assuming that all time slots are equal, the Eq. 12 minus the Eq.13 is the time that NSS-CS saved in WBAN.

$$
\Delta T=(n-k) \cdot S-k \cdot \widetilde{C}_{t}
$$

The time saving $\Delta T$ is related to the number $k$ of nodes that collected abnormal signals and the average time $\widetilde{C}_{t}$ of compression and classification. The $k$ reflects the network load, and $\widetilde{C}_{t}$ is related to $C R$. The delay comparison chart of NSS-CS $(C R)$, traditional TDMA and BCMAC protocol in different $C R$ value is shown in Fig.5.

When $0<k \leq 2$ and $k \geq 14$ under different $C R$, such as the $C R=0,0.1$, or 0.3 . The delay of NSS-CS is larger than the traditional TDMA and BCMAC protocol, but when $C R$ is 0.5 , the delay of NSS-CS is smaller than the traditional TDMA, BCMAC protocol, because when $C R$ is $0,0.1$, or 
0.3 , the time which is spent in compressing and classifying data is more than in transmitting and receiving data. So reducing the data dimension can effectively improve the speed of signal classification and reduce the delay. When $2<k<14$, the delay of NSS-CS are smaller than the traditional TDMA and BCMAC protocol, so the NSS-CS is suitable to be applied in WBAN where the physiological signals are usually in the stable range.

\section{Conclusions}

Signals collected by nodes are usually in the normal state in WBAN. This paper proposes a new node sleep strategy based on CS theory and SRC (NSS-CS) to save energy and achieved the compression and classification of the signal in WBAN. The over-complete dictionary is constructed by physiological signals in the database which are marked and classified. The dimension of the dictionary and the test sample are reduced by the CS theory for reducing delay of the classification and transmission. The test sample is recognized by SRC to stop nodes transmit the normal signal to the center node and switched the node into sleep state, thus the amount of data transmission is reduced and sleep time of nodes is prolonged, so the node energy consumption is lower. Theoretical analysis and simulation results show that compared with traditional TDMA, BCMAC protocol, NSS-CS can effectively reduce power consumption and node delay, and is suitable to be applied in WBAN where signals are usually in the normal range.

\section{Acknowledgements}

This work is supported by Natural Science Fund of Hubei Province (2015CFC802), Research and Development Project of Xiangyang ([2014]12), Open Fund of Collaborative Innovation Center of Hubei Province for Auto Parts Manufacturing Equipment Digitization (hbuascic2014017), Open Fund of "Mechanical and Electrical Automobile" Characteristic and Preponderant Subject Groups of Hubei Province (XKQ2016022), and Research Fund for Doctoral Program of Hubei University of Arts and Science (2013B005).

\section{References}

[1] Jing Liu, Ming Li, Bin Yuan, Wenlong Liu: A novel energy efficient mac protocol for wireless body area network. China Communications, Vol.12, No.2, p. 11-20 (2015).

[2] Candès E J: The restricted isometry property and its implications for compressed sensing. Comptes Rendus Mathematique, Vol.346, No.9, p. 589-592 (2008).

[3] Mi Zhang, Alexander A. Sawchuk: Human daily activity recognition with sparse representation using wearable sensors. IEEE Journal of Biomedical and Health Informatics, Vol.17, No.3, p. 553-560 (2013).

[4] John Wright, Allen Y. Yang, Arvind Ganesh, S. Shankar Sasty, Yi Ma: Robust face recognition via sparse representation. IEEE Transactions on Pattern Analysis and Machine Intelligence, Vol.31, No.2, p. 210-227 (2009).

[5] Dongmei Yan, Jinkuan Wang: Sensor Scheduling Algorithm Target Tracking-Oriented. Wireless Sensor Network, Vol.3, No.8, p. 295-299 (2011).

[6] Candes E J, Tao T: Decoding by linear programming. IEEE Transactions on Information Theory, Vol.51, No.12, p. 4203-4215 (2005).

[7] Eftekhari A, Yap H L, Rozell C J, Wakin M B: The restricted isometry property for random block diagonal matrices. Applied and Computational Harmonic Analysis, Vol.38, No.1, p. 1-31 (2015).

[8] Laska J, Kirolos S, Massoud Y, Baraniuk R, Gilbert A, Iwen M, Strauss M: Random sampling for analog-to-information conversion of wideband signals. IEEE Dallas/CAS Workshop on Design, Applications, Integration and Software, Washington D.C, p. 119-122 (2006).

[9] Kasu S R, Bellana S K, and Kumar C: A binary countdown medium access control protocol scheme for wireless sensor networks. Proceedings of the 10th International Conference on 
Information Technology, Rourkela, Orissa, India, p.122-126 (2007). 